\title{
Characterization of Piezoresistive Sensors of Graphite on Paper Substrate
}

\author{
Luiz Antonio Rasia, $\mathrm{PhD}^{1}$, Patricia Carolina Pedrali, $\mathrm{MS}^{2}$, and Antonio Carlos Valdiero, $\mathrm{PhD}^{1}$ \\ ${ }^{1}$ UNIJUI/DCEEng, Brazil, rasia@unijui.edu.br,valdiero@unijui.edu.br \\ ${ }^{2}$ UNIJUI/CAMPUS PANAMBI, Brazil, patricia.pedrali@unijui.edu.br
}

\begin{abstract}
This article describes the development of piezoresistive sensor elements using exfoliating graphite deposited by direct technique on paper polymeric substrate. We have chosen the paper substrate as a basic material to explore the physical effects of graphite as sensor element. As our first research paper we use to build a force sensor device based on the theory of small deflections. This work shows the feasibility of producing low-cost piezoresistive sensing elements using an allotropic form of carbon alternatively to the traditional processes of manufacturing piezoresistors in silicon substrates. The micro fabrication processes using silicon is very time consuming and expensive involving sophisticated equipment and clean rooms. We are interested in developing a new technology for the production of MicroElectroMechanical Systems (MEMS) with minimal cost but using the typical criteria of the integrated circuit micro fabrication processes.
\end{abstract}

Keywords-Piezoresistive sensor, Graphite sensor, Cellulose substract.

\section{INTRODUCTION}

The graphite has been investigated and used in a wide range of applications due to its excellent mechanical, electrical and thermal properties. This article shows the mathematical modeling, using the theory of small deflections, to characterize the properties of thin graphite films [1] [2] [3] [4]. In this characterization the sensors were deposited, by process known as GOP - Graphite on Paper and encapsulated with epoxy adhesive. The electrical contacts were made using thin copper wires.

Every pencil contains a writing core (or "lead") which is made from a mixture of graphite, wax, and clay. The hardness of the lead depends on the amount of graphite and clay. The more graphite it contains, the softer and thicker it is. Pencil hardness is graded in twenty degrees ranging from $9 \mathrm{H}$ (hardest) to $9 \mathrm{xxB}$ (softest). When using pencils, different types of strokes are produced depending on the pencil's hardness, its point, and how it is applied to the paper. The dimensions of these traces determine the electrical resistance of the sensor element.

Most pencil manufacturers define their own internal standards for the grades of graphite hardness and the overall quality of the core used. Thus, the choice of one type of pencil or another for the manufacture of sensor elements requires the individual characterization of each sensor element produced.

Digital Object Identifier (DOI): http://dx.doi.org/10.18687/LACCEI2018.1.1.327 ISBN: 978-0-9993443-1-6

ISSN: $2414-6390$
The percentage of carbon in the graphite indicates whether it is softer or harder. Therefore; the subdivisions directly influence the mechanical strength and electrical conductivity of the device to be manufactured.

The conductivity and other physical characteristics of the graphite are due to the arrangement of atoms in the material, forming sheet-like structures attracted by weak bonds. In the "sheets," the atoms are organized in the form of hexagons, similar to that of combs in a beehive, where each carbon atom occupies a vertex, as shown in Fig. 1.

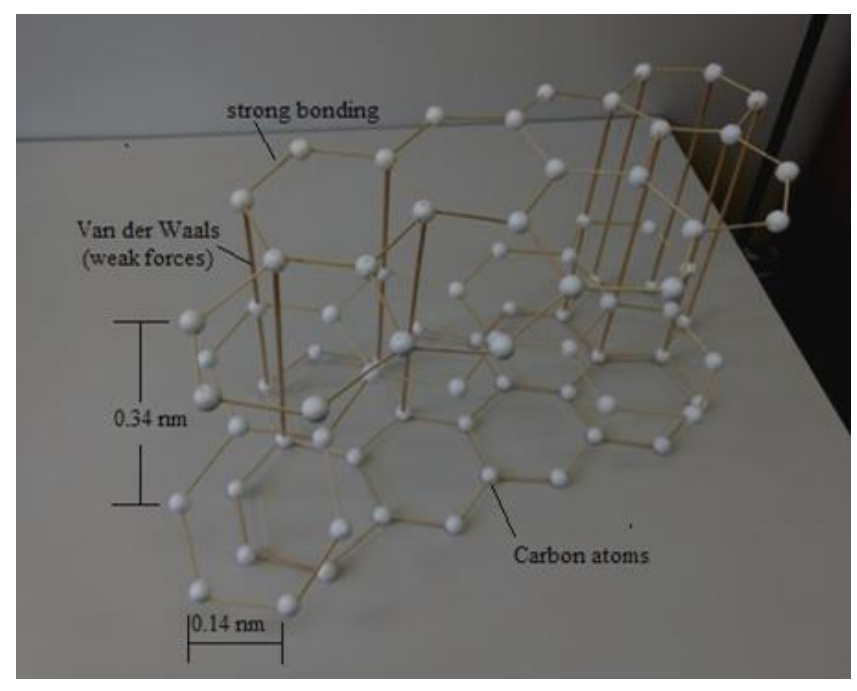

Fig.1. (Graphite Network Structure (Photo Mounted).

Figure 1 shows the network structure of the graphite, where the graphene layers are observed, and weak Van der Waals interactions in addition to the strong covalent bonds. The bonds between the graphite carbons are of the $\mathrm{sp}^{2}$ type, with connections in different planes which gives the graphite the characteristic of conductive material.

When a force is applied to the crimped beam on which graphite has been deposited the carbon atoms are expand or compress, sensibly, inducing a change in electrical resistance. These changes can generate changes in the behavior of a sensor device according to the piezoresistive effect.

Otherwise, the sliding of the layers one on top of the other enables the writing on a paper, due to the small graphite residues that are deposited. This described process is used in this work to make the sensor elements.

$16^{\text {th }}$ LACCEI International Multi-Conference for Engineering, Education, and Technology: "Innovation in Education and Inclusion”, 19-21 July 2018, Lima, Peru. 
We are interested in developing a new technology for the production of MEMS with minimal cost but using the typical criteria of the integrated circuit micro fabrication processes. Thus, we chose the paper substrate as a basic material to exploit the physical effects of graphite as a sensor element in place of silicon. This process involves low cost, few manufacturing steps and is not the use of a clean room.

\section{EXPERIMENTAL AND THEORETICAL STUDY}

In recent years a wide range of paper-based devices and sensors have been developed, including mechanical strain gauges, micro fluidic devices, super capacitors, biological sensors, chemical sensors, infrared radiation detectors and devices MEMS actuators based on paper-polymer composites and others materials [4] [5] [6].

Cellulose is a natural, biodegradable polymer, easy to process in abundance in nature, as illustrate in Fig. 2.

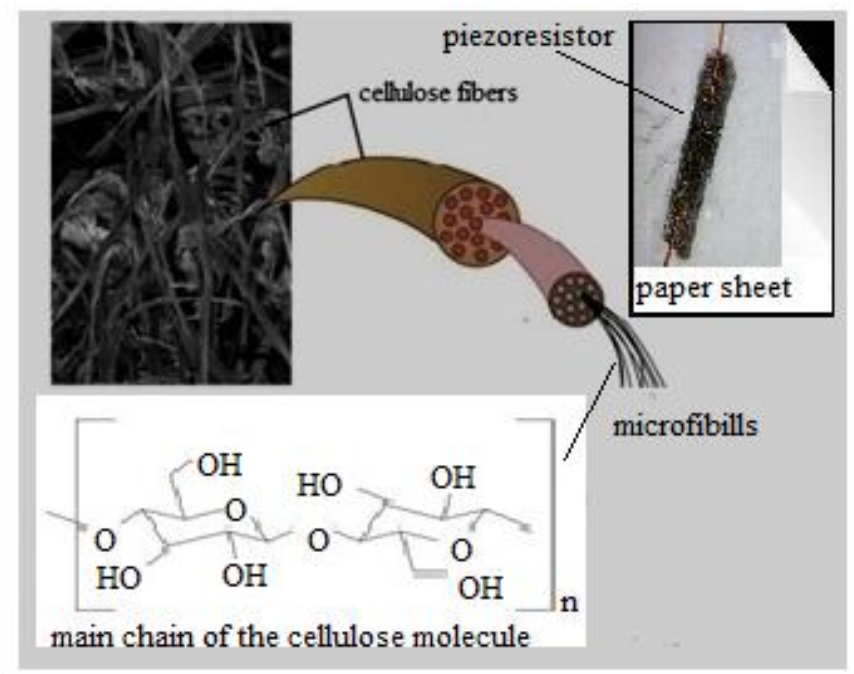

Fig. 2. Structure of a sheet of paper composed of microfibers and details of a piezoresistor of graphite. Adapted from reference [7].

An X-ray diffraction analysis shows that the structure of the cellulose crystal is in the planes (110) and (200), respectively, according to [7]. However, the fibers can be functionalized so that many of their properties are adapted to industrial requirements.

There is more cellulose in the biosphere than any other substance. Its primary structure is simple: a long chain of glucose units attached together by $\beta(1,4)$ linkages. It is the ability of these chains to hydrogen-bond together into fibres (microfibrils) that gives cellulose its unique properties of mechanical strength and chemical stability [7][8].

Cellulose microfibrils contain two crystalline forms, cellulose I $\alpha$ and I $\beta$, in which the chains are packed slightly differently. As hydrogen bonds are different in each shape, reflecting on different physico-mechanical properties.
Cellulose I $\alpha$ or I $\beta$ forms the core of each crystalline unit, in the microfibrils from higher plants, but at the surface there are chains that do not conform to either of these crystalline allomorphs. The monoclinic $\mathrm{I} \beta$ allomorph is the most thermodynamically stable form [8].

Cellulose has the ability to allow liquid substances to pass through its hydrophilic matrix without external forces acting and its derivatives have been used in the most varied segments of science and technology, and especially in the area of electronics. Cellulose also finds its application in the pharmaceutical industry as inactive filler in tablets and in the food processing industry as thickener and stabilizer [6] [7].

This work shows the feasibility of producing inexpensive piezoresistive sensor elements using an allotrope form of carbon in paper substrates instead of the traditional piezoresistive manufacturing processes on silicon substrates.

\section{A. Piezoresistive Effect}

The piezoresistive effect in thin films of graphite on paper substrate is defined by equation (1).

$\frac{\Delta \rho_{i j}}{\rho}=\pi_{\pi i j k l} T_{k l}$

Where, $\pi_{\pi i j k l}$, is the piezoresistive coefficient tensor, an intrinsic property of the material chosen as sensor elements and which can be adjusted by specific doping techniques. The mechanical stress in the structure of the material is given by, $T_{k l}$, being, $\frac{\Delta \rho_{i j}}{\rho}$, the ratio of the electric resistivity when the material is subjected to external forces of traction or mechanical deformations.

In general, the structures of the films deposited on the substrates have a thickness much smaller than the thickness of the substrate itself, $t_{f}<<t_{s}$, therefore, the mechanical stresses are transmitted integrally from the substrate to the film according to the theory of small deflections.

The piezoresistive effect can be described expressing the change of electrical resistance, $\Delta R$, of macroscopic form in function of mechanical stress, mechanical deformation, $\varepsilon$, and the sensitivity factor or gauge factor, $G F$ given by equation (2),

$G F=\frac{\Delta R}{R \varepsilon}$

The sensitivity factor is dependent on the crystallographic orientation of the material and is related to the piezoresistive coefficient, $\pi_{i j}$, through the Young's modulus, $E$, given by equation (3),

$G F=\pi_{i j} E$ 
The piezoresistive coefficient is an intrinsic property dependent on the orientation of the piezoresistor in relation to the crystal axes, material impurities and temperature. This coefficient in general has a non-linear physical behavior.

The resistance, $R$, a long piezoresistor, shown in Figure 4, can be calculated according to [2] using equation (4),

$$
R=\mathrm{R}_{\theta_{\mathrm{amb}}}+\rho_{0} \pi_{L} \int_{x_{1}}^{x_{2}} T_{L}(x) d x+\rho_{0} \pi_{T} \int_{x_{1}}^{x_{2}} T_{T}(x) d x
$$

Where $\pi_{L}$ and $\pi_{T}$ are the longitudinal and transverse piezoresistive coefficients respectively, $T_{L}(x)$ and $T_{T}(x)$ are the voltages applied along the piezoresistor. $R_{\theta_{a m b}}$ is the initial resistance of the material at room temperature given by Eq. (5) and $\rho_{0}$ is the resistivity and $t$ is the thickness of the deposited material when a mechanical stress is not applied in the structure of the de piezoresistor at room temperature.

$R_{\theta_{a m b}}=\rho_{0} \frac{L}{w . t}$

The Figure 3 shown geometric model of the piezoresistor based on the model given by [2] with details of the sensor element of graphite.

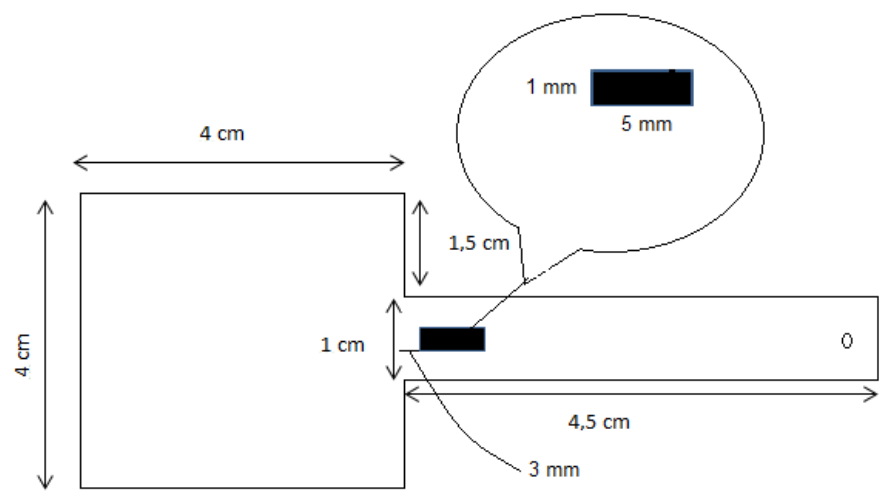

Fig. 3. Piezoresistor design on a crimped beam.

\section{B. Processing Step}

The processing steps for manufacturing the piezoresistores are shown in Fig. 4. These steps involve the choice of substrate cleaning, graphite mechanical exfoliation deposition, contact definition, the welding wire in defined regions, protection of the contacts and encapsulation with epoxy resin.

The encapsulation steps are described by [9] which also show the main results indicating that epoxy resins exhibit good resistance to mechanical and chemical stress. However, their properties depend on the type and conditions of cure.

\begin{tabular}{|c|c|c|}
\hline step $\# 1$ & & $\begin{array}{l}\text { Polymer } \\
\text { substrate }\end{array}$ \\
\hline step $\# 2$ & & $\begin{array}{c}\text { GoP deposition } \\
\text { of graphite }\end{array}$ \\
\hline step $\# 3$ & L & $\begin{array}{c}\text { Contact } \\
\text { definition PAD }\end{array}$ \\
\hline step $\# 4$ & r & $\begin{array}{l}\text { Wire bonding- } \\
\text { copper end } \\
\text { epoxy }\end{array}$ \\
\hline step $\# 5$ & & Packing \\
\hline
\end{tabular}

Fig. 4. Processing steps.

\section{Method of Experimental Characterization}

In this work were made graphite sensors devices type 2B (commercial) deposited on a flexible polymer (paper) and encapsulated with epoxy adhesive as shown in Fig. 5.

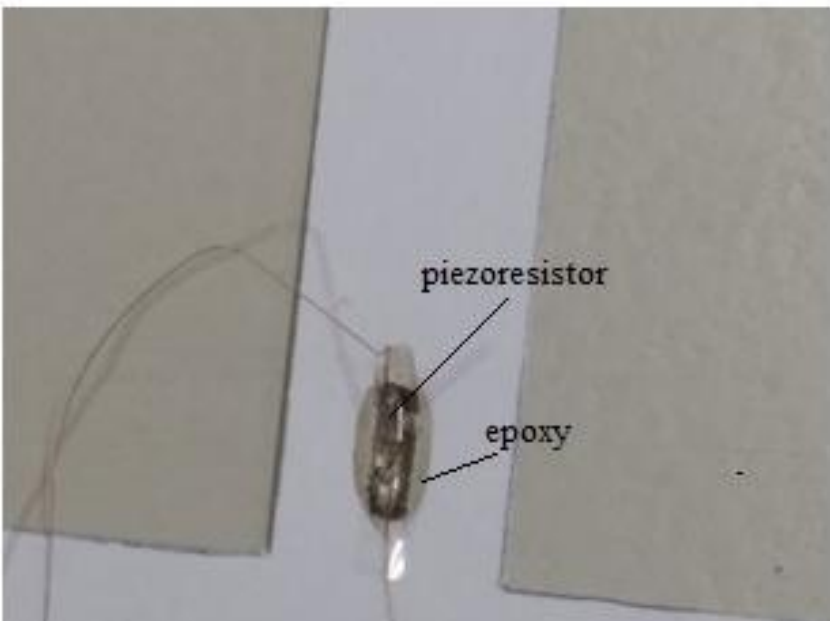

paper substrate

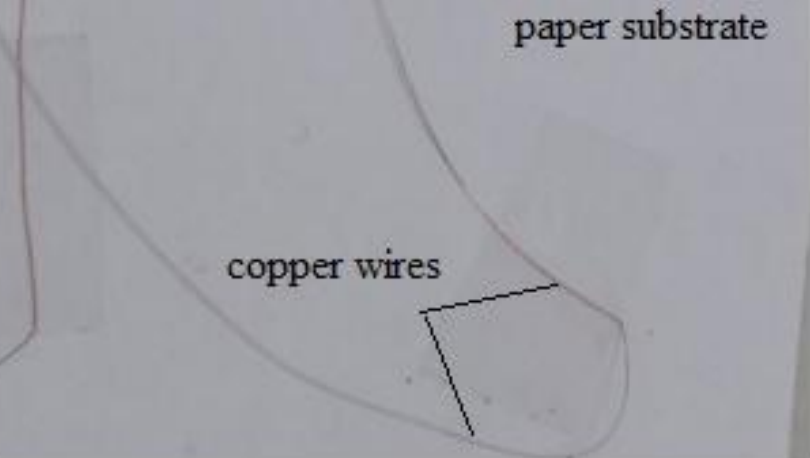

Fig. 5. Photograph of an encapsulated graphite piezoresistor.

$16^{\text {th }}$ LACCEI International Multi-Conference for Engineering, Education, and Technology: "Innovation in Education and Inclusion”, 19-21 July 2018, Lima, Peru. 
The coating of the sensor element through the encapsulation of the graphite film is an essential part of the piezoresistive structure. The encapsulation gives the sensor protection against the action of undesirable environmental factors that may compromise its properties and protect against external agents that may compromise its operation.

The deposition of the epoxy resin on the sensor elements was carried out manually, keeping the quantity and shape constant.

Figure 6 illustrates the deposition process of the thin graphite film prior to the steps of welding the copper wires and the encapsulation. The copper wires were initially attached with adhesive tape to the initial tests of electrical resistance measurements.

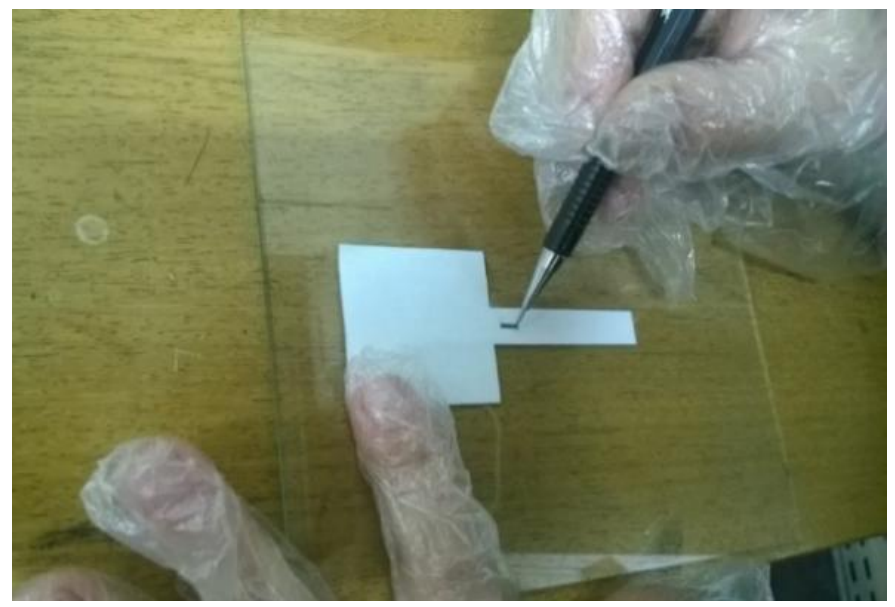

Fig. 6. Photograph of the manual process of deposition of graphite on paper.

The electrical contacts were made with thin copper wires and the resistivity of the graphite deposited on the paper substrate was measured being of the order of $5.3 \times 10^{-5} \Omega . \mathrm{m}$.

The deflection method of a crimped beam at one end illustrated in Fig. 7 is used to obtain and characterize sensor elements [9].

\section{not mechanically stressed}
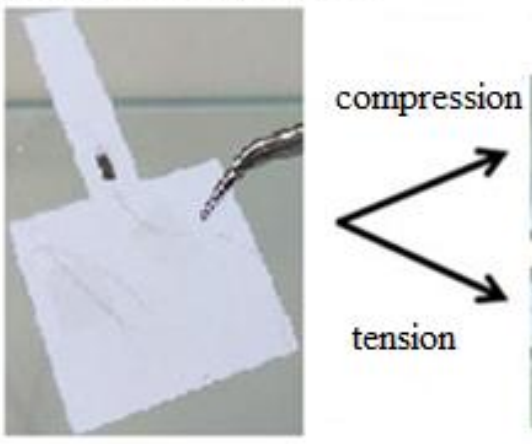

tension

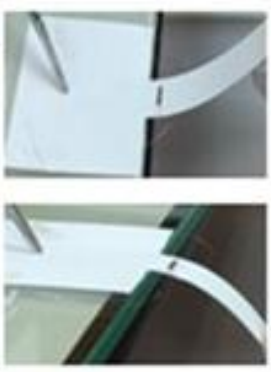

In the cantilever the contact force the mechanical stress, $T$, is related by the modulus of elasticity, $E$, and by the mechanical deformation, $\varepsilon$, and other geometries of the material.

The crimped beam behaves as if it were a spring, where its mechanical rigidity suffers direct dependence of the geometry and the composition of the materials used in its composition. In order for the material to not rupture, the mechanical stress limit used should not exceed the elastic limit of the compounds used according to Hooke's Law.

In the application of mechanical stresses, small weights were used. With masses varying from $0.095 \mathrm{~g}$ to $1.5 \mathrm{~g}$ coupled to the cantilever as shown in Fig. 7. Mathematical equations and models were implemented using MatLab ${ }^{\mathrm{TM}}$ software.

The uniformity of deposition of the film was inspected through a digital microscope model AM-313T and piezoresistance was measured with a multimeter Agilent model 34401A as shown in Fig. 8.

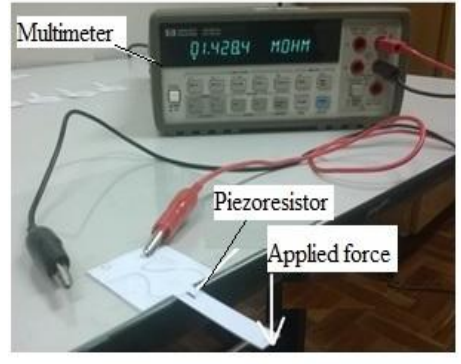

(a)

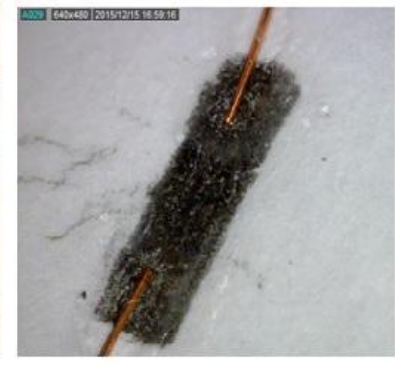

(b)
Fig. 8. (a) Experiment Setup Photography and (b) piezoresistor on paper.

Digital microscopy allows the analysis of the presence or absence of uniformities in the piezoresistive film and the layer thickness of the deposited layer. The existence of these "free graphite samples", as evidenced in the images, result in possible deformations or irregularities formed in the construction process of the piezoresistor, may interfere with the final resistance of the sensor element, the conductivity and the quality of the material produced.

\section{RESULTS AND DISCUSSION}

Figure 9 shows the behavior of the piezoresistor with the longitudinal (a) and transverse (b) mechanical stress applied and compared with the results predicted by the model.

Fig. 7. Illustration of the crimped beam method.

$16^{\text {th }}$ LACCEI International Multi-Conference for Engineering, Education, and Technology: "Innovation in Education and Inclusion", 19-21 July 2018, Lima, Peru. 


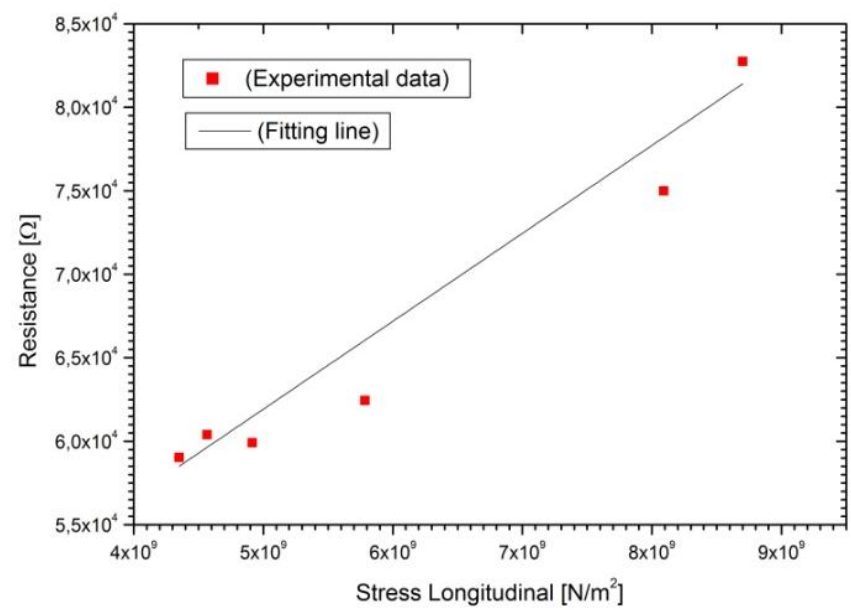

Fig. 9 (a) Piezoresistance versus mechanical stress longitudinal

The results shown in Fig. 9 (a) and Fig. 9 (b) show a linear trend according to the experimental data. However small nonlinearities are observed which are due to the film structure itself and the experimental arrangement used.

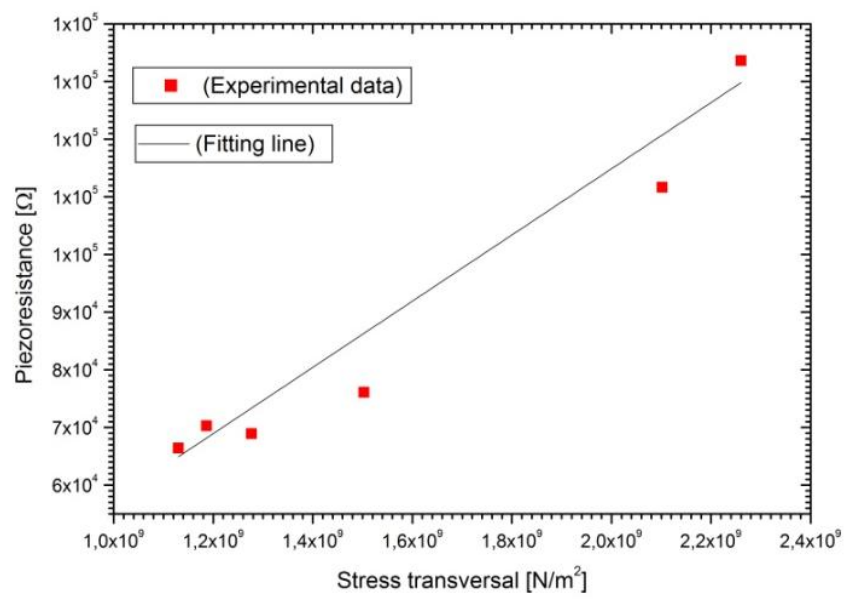

Fig. 9 (b) Piezoresistance versus mechanical stress transversal.

The analysis of the figures shows that the mechanical tension differs by Poisson coefficient in each situation plotted. These figures indicate the use of graphite as sensor element and allow you to extract the piezoresistive coefficients or sensitivity of the material.

The Fig. 10 illustrates the sensitivity of the piezoresistor as a function of the applied mechanical stress.

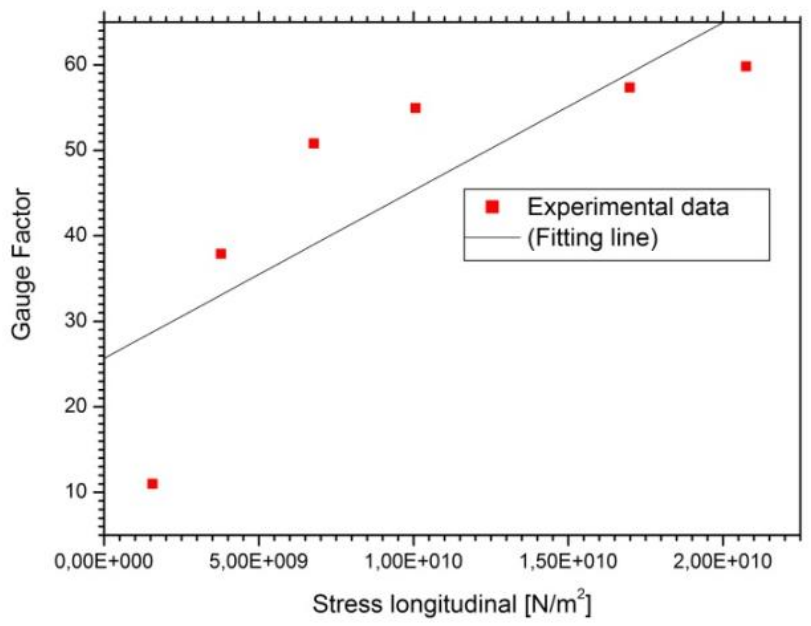

Fig. 10. Trend of the longitudinal sensitivity factor with increased mechanical stress.

Fig. 10 shows that the sensitivity of the piezoresistor follows a linear trend as a function of mechanical stress. This result indicates that even increasing mechanical stress increases GF to the breaking point of the material. This result is an indication that in the medium and for small ranges of measurements the GF remains practically constant. The piezoresistive coefficient obtained is of the order of $9.61 \times 10^{-10} \mathrm{~m}^{2} / \mathrm{N}$.

\section{CONCLUSIONS}

The results of this work show that the average sensitivity of the graphite allows the fabrication of sensor elements using the procedures presented here. The equations that correlate the different physical quantities allow to design a piezoresistor of graphite from pencils through a process of low cost and with a good linearity.

\section{ACKNOWLEDGMENT}

The authors thank the CNPq and FAPERGS for financial assistance and the availability of laboratories UNIJUI.

\section{REFERENCES}

[1] S. Timoschenko., S. Woinowsky-Krieger., Theory of plates and shells, McgrawHill, New York, 2th edition, 2001.

[2] Z. Gniazdowski et. Al., New Approach to Exact Extraction of Piezoresistance Coefficient, Sensors and Actuators A, Vol. 68, pp.329332, 1998

[3] A. Tibrewala, et al., Piezoresistive gauge factor of hydrogenated amorphous carbon films, J. Micromech. Microeng. Vol. 16, n. 6, pp. S75, 2006.

[4] A. J. Gimenez, J. M. Yáñez-limón, J. M. Seminario, Paper-Based Photoelectrical Devices. Journal of Intelligent Material Systems and Structures, pp.1-7,2012 DOI: 10.1177/1045389X12457836.

[5] J. C. Cunningham, P. R. Degregory and R. M. Crooks, New Functionalities for Paper-Based Sensors Lead to Simplified User Operation, Lower Limits of Detection, and New Applications. Annu. Rev.

$16^{\text {th }}$ LACCEI International Multi-Conference for Engineering, Education, and Technology: "Innovation in Education and 
Anal. Chem. 2016. 9:4.1-4.20. DOI: 10.1146/annurev-anchem-071015041605.

[6] J. Kim, S. Yun, Z. Ounaies, Discovery of Cellulose as a Smart Material. Macromolecules 2006, 39 (12), pp. 4202-4206. DOI: $10.1021 / \mathrm{ma} 060261 \mathrm{e}$.

[7] M. K. Suresha, W. Konrad, S. Boris, Paper as a Platform for Sensing Applications and Other Devices: A Review ACS Appl. Mater. Interfaces, pp. 1-65, 2015. DOI: 10.1021/acsami.5b00373.

[8] M. Akerholm, B. Hinterstoisserb and L. Salmen, Characterization of the crystalline structure of cellulose using static and dynamic FT-IR spectroscopy, Carbohydrate Research 339 (2004) pp. 569-578.

[9] R. Berkenbrock, L. A. Rasia, P. C. Pedrali, A. C. Valdiero, Piezoresistive Graphite Sensors Encapsulated With Epoxy ResinBisphenol A (BPA), American Journal of Engineering Research, pp. 148 - 154, 2018.

$16^{\text {th }}$ LACCEI International Multi-Conference for Engineering, Education, and Technology: "Innovation in Education and Inclusion”, 19-21 July 2018, Lima, Peru. 